\title{
The sputum microbiome is distinct between COPD and health, independent of smoking history
}

\author{
Koirobi Haldar ${ }^{1 \dagger}$, Leena George ${ }^{1 \dagger}$, Zhang Wang ${ }^{2}$, Vijay Mistry ${ }^{1}$, Mohammadali Yavari Ramsheh ${ }^{1}$, Robert C. Free ${ }^{1}$, \\ Catherine John ${ }^{3}$, Nicola F. Reeve ${ }^{3}$, Bruce E. Miller ${ }^{4}$, Ruth Tal-Singer ${ }^{4}$, Adam J. Webb $^{5}$, Anthony J. Brookes ${ }^{5}$, \\ Martin D. Tobin ${ }^{3}$, Dave Singh ${ }^{6}$, Gavin C. Donaldson7, Jadwiga A. Wedzicha7, James R. Brown ${ }^{8}$, \\ Michael R. Barer ${ }^{1}$ and Christopher E. Brightling ${ }^{1, *^{*}}$ (i)
}

\begin{abstract}
Background: Airway bacterial dysbiosis is a feature of chronic obstructive pulmonary disease (COPD). However, there is limited comparative data of the lung microbiome between healthy smokers, non-smokers and COPD.

Methods: We compared the 165 rRNA gene-based sputum microbiome generated from pair-ended Illumina sequencing of 124 healthy subjects (28 smokers and 96 non-smokers with normal lung function), with single stable samples from 218 COPD subjects collected from three UK clinical centres as part of the COPDMAP consortium.

Results: In healthy subjects Firmicutes, Bacteroidetes and Actinobacteria were the major phyla constituting 88\% of the total reads, and Streptococcus, Veillonella, Prevotella, Actinomyces and Rothia were the dominant genera.

Haemophilus formed only $3 \%$ of the healthy microbiome. In contrast, Proteobacteria was the most dominant phylum accounting for 50\% of the microbiome in COPD subjects, with Haemophilus and Moraxella at genus level contributing 25 and 3\% respectively. There were no differences in the microbiome profile within healthy and COPD subgroups when stratified based on smoking history. Principal coordinate analysis on operational taxonomic units showed two distinct clusters, representative of healthy and COPD subjects (PERMANOVA, $p=0.001$ ).

Conclusion: The healthy and COPD sputum microbiomes are distinct and independent of smoking history. Our results underline the important role for Gammaproteobacteria in COPD.
\end{abstract}

Keywords: COPD, Healthy airway, Microbiome, Haemophilus, Proteobacteria

\section{Background}

Chronic obstructive pulmonary disease (COPD) is characterised by inflammation and irreversible airflow obstruction. Before the advent of culture-independent DNA profiling methods, the healthy lung was deemed a

\footnotetext{
* Correspondence: ceb17@le.ac.uk

${ }^{\dagger}$ Koirobi Haldar and Leena George contributed equally to this work. 'Institute for Lung Health, NIHR, BRC, Department of Respiratory Sciences, College of Life Sciences, University of Leicester, Leicester LE1 7RH, UK ${ }^{9}$ Institute for Lung Health, University Hospitals of Leicester, Groby Road, Leicester LE3 9QP, UK

Full list of author information is available at the end of the article
}

sterile niche while COPD samples would frequently culture Haemophilus influenzae, Streptococcus pneumoniae, Moraxella catarrhalis and Pseudomonas aeruginosa. It is considered that pathogenic bacteria gain a foothold in damaged airways contributing to further lung pathology through release of noxious bacterial products and provocation of host inflammation $[1,2]$.

In recent years, use of high-throughput $16 \mathrm{~S}$ rRNA gene based sequencing has demonstrated that rich, complex bacterial communities exist in the airways of both health and COPD, with overlapping bacterial 
composition observed [3, 4]. In COPD aerobic, facultative and anaerobic organisms colonise the airways $[3,5]$, with Proteobacteria and Firmicutes being the two major phyla reported in the microbiome and Haemophilus and Streptococcus, the respective dominant genera $[3,4,6$, 7]. At exacerbation, shifts in bacterial composition, characterised by a relative increase in Proteobacteria that falls in response to antibiotics has been observed, suggesting an association with the aetiology of COPD exacerbations [8, 9]. Furthermore, the ratio of Gammaproteobacteria to Firmicutes identifies the subgroup with dynamic changes in their microbiome during exacerbation, suggesting a potential use of this ratio as a biomarker for targeting antimicrobial treatment [10].

While Proteobacteria have been associated with COPD exacerbation events, the role of the microbiome in the stable state and important differences in composition with health are unclear $[3,4,6]$. Furthermore, changes in the microbiome that may associate with development of COPD in smokers are unclear. Differences have been shown in the nasal and oropharyngeal microbiome between smokers and non-smokers [11] but microbiome data from healthy smokers and non-smokers is limited making the findings inconclusive to contextualize the pathological basis of the observations in COPD.

To address this, we have used sputum collected from a substantial number of well characterised healthy volunteers to investigate the impact of smoking on healthy lower airway microbiome; to explore if there are differences in microbiome between health and COPD and its association with smoking in health.

\section{Methods}

\section{Subjects and study samples}

Healthy volunteers $(n=251)$ (excluded participants with asthma, COPD or bronchiectasis) were selected from the Extended Cohort for E-health, Environment and DNA (EXCEED) cohort and assessed at a single centre, Glenfield Hospital, Leicester. Participants with $\geq 10$ pack year (PY) smoking were grouped as healthy smokers and remaining as occasional / never smokers (<10 PY) [12]. Participant demographics and clinical characteristics including lung function, blood and sputum cell differentials were recorded. The comparator COPD group comprised of a single stable (non-exacerbation) visit sputum collected from 218 subjects (included ex- and current smokers) at three centres, Leicester, London and Manchester, as part of the COPDMAP consortium (www.copdmap.org; NCT01620645) [13]. All the participants in both studies had a minimum of 6 weeks antibiotic free period before their sample collection. Sputum induction was undertaken if an adequate spontaneous sample was not produced. In most of the healthy participants induced sputum was collected. Both cohort studies had ethical approval and all subjects gave written informed consent before the performance of any studyrelated assessments.

All patients provided written informed consent using protocols approved by the local Ethics Committees at each site (London- 11/L0/1630; Manchester- 10/H/ 1003/108; Leicester- 07/H0406/157).

\section{$16 \mathrm{~S}$ rRNA gene sequencing}

As a part of standard routine, for both studies, sputum plugs were separated from the salivary contents to minimise the oral bacterial contamination in samples. Samples with only salivary contents were not processed for microbial work. Sputum plugs, were stored $\left(-80^{\circ} \mathrm{C}\right)$ and processed for high-throughput sequencing similar to the COPD cohort samples [13]. Briefly, bacterial genomic DNA was extracted from the homogenised $(0.1 \%$ dithiothreitol) plugs using the lysozyme-based lysis procedure from Qiagen DNA Mini kit (Qiagen, CA, USA) as per manufacturer's protocol. Out of 251 samples only 137 had adequate DNA concentration for microbiome analysis. Amplicon library was generated utilizing 28 PCR cycles and targeting the $\mathrm{V} 4$ hypervariable region of the 16S rRNA gene with 515F: 5' GTGCCAGCMGCC GCGGTAA3', 806R: 5'GGACTACHVGGGTWTCTAAT3' primers, including Illumina sequencing adapters and a 12 bp Golay barcode sequence attached to forward primer. Pair-ended sequencing was performed using multiplex libraries on the Illumina MiSeq platform. Sequencing run included a commercial mock community DNA (ZymoBIOMICS microbial DNA standard) as a positive control and DNA extraction negative control (each batch of healthy sample DNA extraction included a DNA extraction negative control and a single pooled aliquot was prepared from all of these controls for sequencing) and PCR negative control for reagent contamination check. PCR negative controls didn't produce any reads and DNA extraction negative control only produced 8 raw reads and therefore were not processed further.

COPDMAP single stable samples, utilized here for comparative analysis with healthy samples, had their sequence data generated as part of that study along with appropriate sequencing controls [13] and the sequence data are deposited at the National Centre for Biotechnology Information Sequence Read Archive (SRP102480).

\section{Microbiome analysis}

Reads were processed using QIIME pipeline version 1.9.1 [14] after adaptor trimming and removing low quality reads with Trimmomatic 0.36 [14, 15]. Pairended sequences were joined using fastq-join with a minimum $10 \mathrm{bp}$ overlap [16]. The joined sequences were filtered with a Phred score $\geq 20$ and processed to remove 
contaminating sequences and chimeras using UCHIME [17]. Sequence reads are deposited at the National Centre for Biotechnology Information (SRA accession: PRJNA491861) https://www.ncbi.nlm.nih.gov/bioproject/ PRJNA491861/.

Based on rarefaction curves, healthy and COPDMAP samples, were normalised to 11,000 reads sequencing depth leaving 124 samples in healthy and 218 samples in COPDMAP for microbiome analysis. Operational taxonomic units were generated at $97 \%$ sequence identity using close reference (OTU) method and Greengenes database (version 13_8) and assigned taxonomic identities with the RDP classifier [18]. Alpha diversity index was generated based on the number of OTUs (observed_OTUs and Chao1-richness measure) and their distribution (Shannon index- diversity measure) within a sample. Beta diversity index was based on weighted UniFrac distance measure [19] (phylogenetic distance-based) between OTUs present in each sample and visualized using PCoA plots.

\section{Microbial function prediction through 16S rRNA gene sequences}

Predictive microbial functional profile was generated using the PICRUSt software (v1.0.0) which infers the pathway content of the microbiome by assigning bacterial functional genes for the OTUs, normalised for $16 \mathrm{~S}$ rRNA gene copy number, using the Kyoto Encyclopaedia of Genes and Genomes (KEGG) database [20]. Statistical analysis and visualisation plots were generated using Statistical Analysis of Metagenomic Profiles (STAMP) software [21].

\section{Statistical analysis}

Univariate statistical analyses were performed using GraphPad Prism (Version 7, San Diego, CA). Parametric and nonparametric data are presented as mean (SEM) and median (interquartile range) respectively. Most of the dataset did not meet the normal distribution criteria (Shapiro-wilk test). Therefore, non-parametric MannWhitney test and Kruskal-Wallis (KW) test were performed for between-group comparison of two or greater than two groups respectively. For the KW test, Dunn's multiple comparison test was used to determine statistical significance of pair-wise comparisons. For parametric data, equivalent parametric statistical tests were performed. For categorical data, Chi-square test was performed.

PERMANOVA [22] was performed to test if the overall healthy microbiome was different to COPD using weighted UniFrac distance measure as input. The individual OTUs contributing to differences in health and COPD were identified by performing a non-parametric group-significance test and a corrected $p$-value of $\leq 0.05$ for multiple testing conditions was considered statistically significant. To rank the discriminating taxonomic groups between health and COPD, linear discriminant analysis (LDA) effect size (LEfSe) analysis was performed [23], which detects taxonomies with differential abundance $(p \leq 0.05)$ using the KW test and then assigns a LDA based effect size score. A threshold of $\geq 3.6$ LDA score was used instead of default value of 2 to feature the most discriminant bacterial groups [24] . To determine if any individual microbiome constituents were associated with any subject metadata, MaAsLin was performed [25] and Benjamini-Hochberg (FDR) corrected $q<0.05$ (FDR-adjusted $P$ value) for multiple comparisons was considered statistically significant.

\section{Results}

Table 1 summarizes the demographics and clinical features of the healthy and COPD cohorts (refer to 'Methods' section for more details on both the cohort selection criteria).

\section{Healthy sputum microbiome}

A total of 1424 OTUs at $97 \%$ sequence identity were observed in 124 healthy samples after rarefaction. Most OTUs belonged to Firmicutes $(55 \% \pm 13 \%)$ followed by Bacteroidetes $(21 \% \pm 11 \%)$ and Actinobacteria $(12 \% \pm 6 \%)$ phyla (Fig. 1a). Streptococcus $(30 \% \pm 13 \%)$ was the most abundant genus followed by Veillonella $(17 \% \pm 9 \%)$, Prevotella $(16 \% \pm 10 \%)$, Actinomyces $(6 \% \pm 5 \%)$, Rothia $(5 \% \pm 4 \%)$ and Granulicatella $(3 \% \pm 3 \%)$ (Fig. 1b). The Proteobacteria phylum constituted $7 \%( \pm 7 \%)$ of the bacterial community with Haemophilus $(3 \% \pm 5 \%)$ as its dominant genus.

Compared to participants with a smoking history of $<$ 10 pack year (PY), the subgroup with $\geq 10 \mathrm{PY}$ showed a higher proportion of Firmicutes and a lower proportion of Bacteroidetes at phylum level (Fig. 1a); followed by higher Streptococcus and lower Prevotella at the genus level but these differences did not reach statistical significance (Fig. 1b). Beta diversity based principal coordinate analysis (PCoA) plots did not reveal distinct microbiome clusters for the two smoking subgroups (Fig. 1c). Shannon index was higher $(p<0.01)$ in healthy subjects with $<10$ PY smoking history compared with $\geq 10 \mathrm{PY}$, suggesting a more diverse microbiome in the former (Fig. 1d) but there was no significant difference in observed_OTUs or Chao1 index.

\section{COPDMAP stable sputum microbiome}

A total of 2329 OTUs at $97 \%$ sequence identity were observed in 124 stable COPD samples after rarefaction. The Proteobacteria $(51 \% \pm 12 \%)$ phylum constituted half of the COPD microbiome with Haemophilus $(25 \% \pm 8 \%)$ as its most abundant genus followed by Erwinia $(7 \% \pm$ 
Table 1 Healthy and COPD subject characteristics

\begin{tabular}{|c|c|c|c|c|c|}
\hline & Healthy control $(n=124)$ & $\begin{array}{l}\text { Healthy control } \\
P Y \geq 10(n=28)\end{array}$ & $\begin{array}{l}\text { Healthy control } \\
\mathrm{PY}<10(n=96)\end{array}$ & $\operatorname{COPD}(n=218)$ & $p$-value ${ }^{*}$ \\
\hline Gender female (n) & 73 & 15 & 58 & 60 & $<0.0001$ \\
\hline Age (years) & $61(54,67)$ & $65(58,69)$ & $59(53,67)$ & $69(64,74)$ & $<0.0001$ \\
\hline $\mathrm{BMI}$ & $27 \cdot 7(24 \cdot 3,31 \cdot 2)$ & $29 \cdot 3(25 \cdot 8,34 \cdot 4)$ & $27 \cdot 3(24 \cdot 2,29 \cdot 9)$ & $26 \cdot 7(23 \cdot 32,30 \cdot 06)$ & 0.0325 \\
\hline Smoking history (Pack years) & $0(0,8)$ & $29(17 \cdot 3,45 \cdot 8)$ & $0(0,0)$ & $46(34,64)$ & $<0.0001$ \\
\hline MRC Dyspnoea scale & $0(0,1)$ & $0(0,1)$ & $0(0,1)$ & $2(1,2)$ & $<0.0001$ \\
\hline VAS dyspnoea (mm scale) & $0(0,2)$ & $0(0,3.5)$ & $0(0,2)$ & $30(15,49)$ & $<0.0001$ \\
\hline Post BD FEV1 (L) & $2.87(2.43,3.37)$ & $2 \cdot 82(2 \cdot 33,3 \cdot 24)$ & $2 \cdot 88(2 \cdot 46,3 \cdot 40)$ & $1.45(1.03,1 \cdot 86)$ & $<0.0001$ \\
\hline Post BD FEV1\% Predicted & $109(100,118)$ & $105(92,118)$ & $110(100,118)$ & $57(42,69)$ & $<0.0001$ \\
\hline Post BD FEV1/FVC \% & $80(77,83)$ & $78(75,82)$ & $80(78,83)$ & $51(41,59)$ & $<0.0001$ \\
\hline Blood White cell count $\left(\times 10^{\wedge} 9 / L\right)$ & $5 \cdot 8(4 \cdot 9,7 \cdot 3)$ & $6 \cdot 4(5 \cdot 1,7 \cdot 5)$ & $5 \cdot 7(4 \cdot 8,7 \cdot 25)$ & $7 \cdot 2(6 \cdot 3,8 \cdot 8)$ & $<0.0001$ \\
\hline Blood Neutrophil count $\left(\times 10^{\wedge} 9 / \mathrm{L}\right)$ & $3 \cdot 3(2 \cdot 7,4 \cdot 2)$ & $3 \cdot 8(2 \cdot 8,4 \cdot 3)$ & $3 \cdot 2(2 \cdot 6,4 \cdot 1)$ & $4.7(3.9,5.7)$ & $<0.0001$ \\
\hline Blood Eosinophil count $(\times 10 \wedge 9 / L)$ & $0.14(0.09,0.23)$ & $0.18(0.11,0.25)$ & $0.14(0.09,0.22)$ & $0.21(0.14,0.27)$ & $<0.0001$ \\
\hline Sputum TCC $\left(\times 10^{6} / \mathrm{g}\right)$ & $2 \cdot 4(1 \cdot 1,4 \cdot 2)$ & $3.19(1.6,5.9)$ & $2 \cdot 2(1,3 \cdot 6)$ & $10 \cdot 5(4 \cdot 7,13 \cdot 0)$ & $<0.0001$ \\
\hline Sputum eosinophils \% & $0.25(0,0.75)$ & $0.25(0.18,2 \cdot 38)$ & $0.25(0,0.75)$ & $0.75(0,2)$ & 0.0029 \\
\hline Sputum neutrophil (\%) & $60(43,74)$ & $65(42,75)$ & $56(43,73)$ & $75(40,89)$ & 0.0011 \\
\hline
\end{tabular}

Data is expressed as median (IQR). BMI body mass index, Sputum TCC sputum total cell count, FEV1 forced expiratory volume in $1 \mathrm{~s}$, Post BD FEV1 spirometry recording post bronchodilator; FVC forced vital capacity. ${ }^{*}=$ represents Kruskal-Wallis test comparing between healthy $(P Y \geq 10)$, healthy $(P Y<10)$ and COPD

$3 \%)$, Cronobacter $(6 \% \pm 2 \%)$ and Moraxella $(3 \% \pm 7 \%)$ (Fig. 2a and b). The two other abundant phyla were Firmicutes $(29 \% \pm 9 \%)$ and Bacteroidetes $(16 \% \pm 5 \%)$ dominated respectively by Veillonella $(16 \% \pm 9 \%)$, Granulicatella $(7 \% \pm 3 \%)$ and Streptococcus $(5 \% \pm 2 \%)$ and by Prevotella $(14 \% \pm 5 \%)$ at the genus level (Fig. 2a and $\mathrm{b}$ ).

COPD ex-smokers and current smokers showed similar bacterial composition at phylum and genus level (Fig. $2 a$ and $b$ ) and no significant difference in their microbial diversity measurements (Fig. $2 \mathrm{c}$ and $\mathrm{d}$ ). There was no significant difference in the microbiome profile of the COPD samples from the three centres (efigure 1).

\section{Healthy vs COPD microbiome}

In the healthy microbiome Streptococcus (28\%) from the Firmicutes (55\%) phylum was the predominant constituent, while Haemophilus (3\%) from Proteobacteria (7\%) was present at low levels. In contrast, for the COPD cohort Haemophilus (25\%) was the most dominant genus with a low proportion of Streptococcus (5\%) observed (Fig. 3a and b). PCoA analysis showed distinct clusters of healthy and COPD subjects with significant difference in their microbiome by permutation multivariate analysis of variance (PERMANOVA $p=0.01$ ) (Fig. 2c). COPD samples had more OTUs identified compared to healthy individuals, with significantly higher alpha diversity indices (Fig. 3d). Linear discriminant effect size (LEfSe) analysis revealed a higher abundance of Gammaproteobacteria species and lower proportion of Firmicutes, Bacteroidetes and Actinobacteria taxa to be the major contributors in differentiating COPD from health (Fig. 4). OTUs differentiating the two groups are presented in Table 2.

Multivariate analysis by linear models (MaAsLin) analysis detected no significant association either in COPD or in health between the bacterial groups and clinical characteristics related to smoking, lung function and symptom score.

Phylogenetic Investigation of Communities by Reconstruction of Unobserved States (PICRUSt) analysis was performed to predict functional gene content from the 16S rRNA gene content. PCoA plots based on this analysis showed distinct clusters of COPD and health (Fig. 5a). Functional genes associated with Bacterial motility proteins, lipopolysaccharide biosynthesis, $A B C$ transporters and secretion systems were in higher proportion in COPD while metabolic pathways were more abundant in healthy subjects (Fig. 5b).

\section{Discussion}

Our study is the largest to compare the sputum microbiome between health and COPD and clear differences between these groups were identified. Firmicutes, Bacteroidetes and Actinobacteria comprised $88 \%$ of the sputum microbiome in healthy participants, with Streptococcus, Prevotella and Veillonella as the dominant genera. Haemophilus, the dominant genus in COPD was present in health at a significantly lower proportion. Healthy smokers with $\geq 10$ PY smoking history showed a trend towards a higher ratio of Streptococcus to Prevotella. 


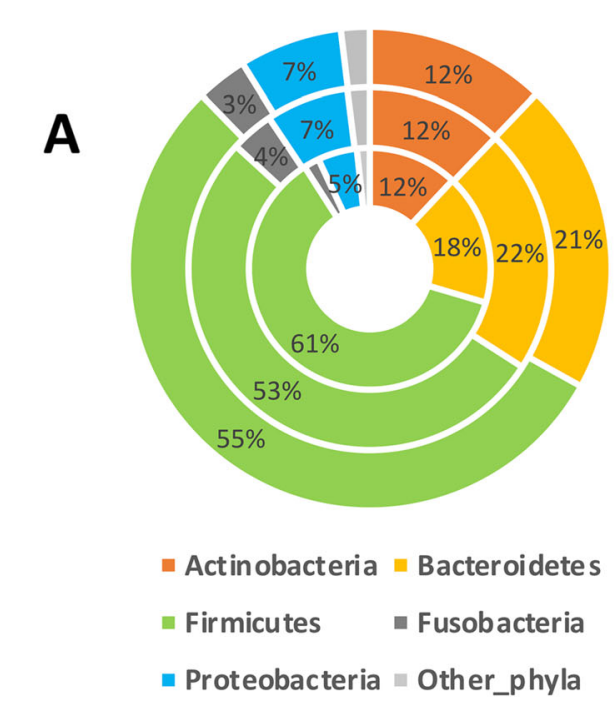

C

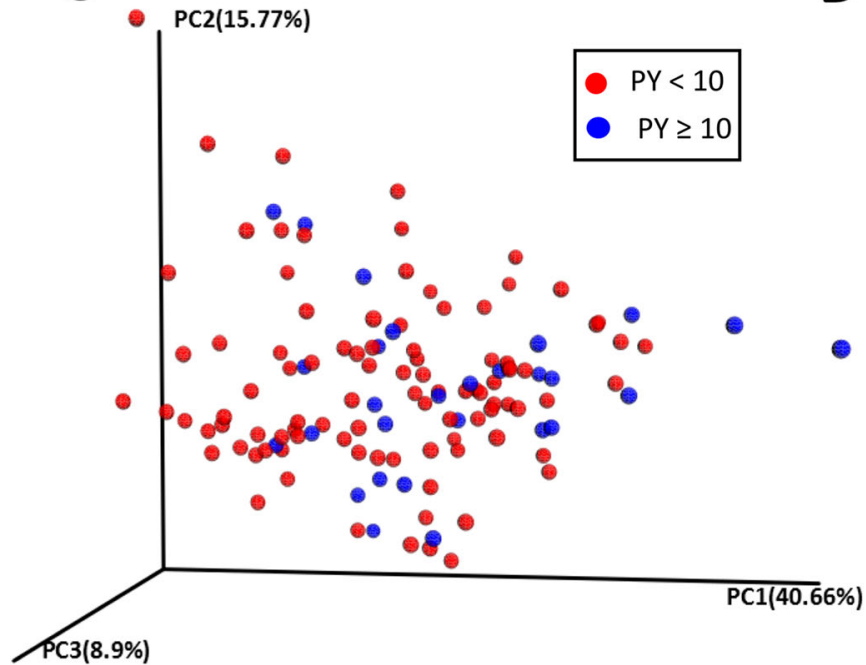

D
B

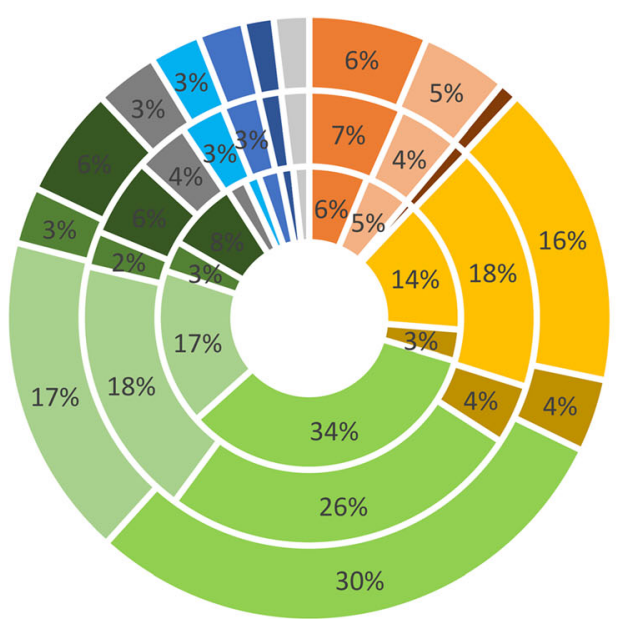

\section{- Actinomyces}

- Actin obacteria_others

Rothia

- Bacteroidetes_others

- Veillonella

- Firmicutes_others

- Haemophilus

- Proteobacteria_others
- Prevote lla

- Streptococcus

- Granulicatella

- Fusobacteria_others

- Unclassified_Ne isseriaceae

Others

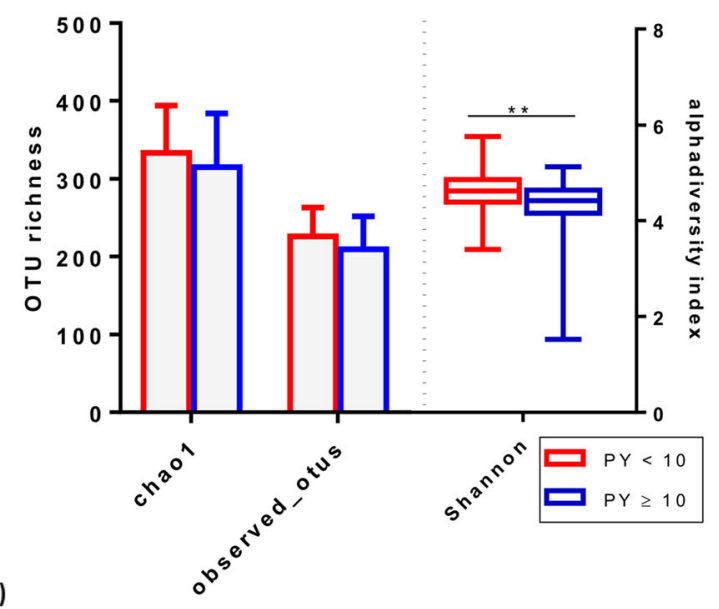

Fig. 1 Microbiome profile of Healthy volunteers based on smoking pack year history. a Relative abundance of major phyla between all healthy $(n=124)$ represented in the outer ring followed by healthy $<10$ PY smoking history subgroup $(n=96)$ in the middle ring and innermost ring representing healthy $\geq 10 \mathrm{PY}$ history subgroup $(n=28)$. b Relative abundance of major genera between all healthy $(n=124)$ represented in the outer ring followed by healthy $<10$ PY $(n=96)$ in the middle ring and innermost ring representing healthy $\geq 10$ PY history $(n=28)$. c Principal coordinate analysis (PCOA) analysis of weighted unifrac distance measures relative to pack year history. $\mathbf{d}$ Alpha diversity indices comparison between. < 10 PY and $\geq 10$ PY smoking sub-groups. Chao1 and observed_otus are represented as bar chart as mean and standard deviation; Shannon index is represented by box whisker plot showing median, interquartile range and minimum and maximum. ${ }^{* *}$. $P<0.01$ 


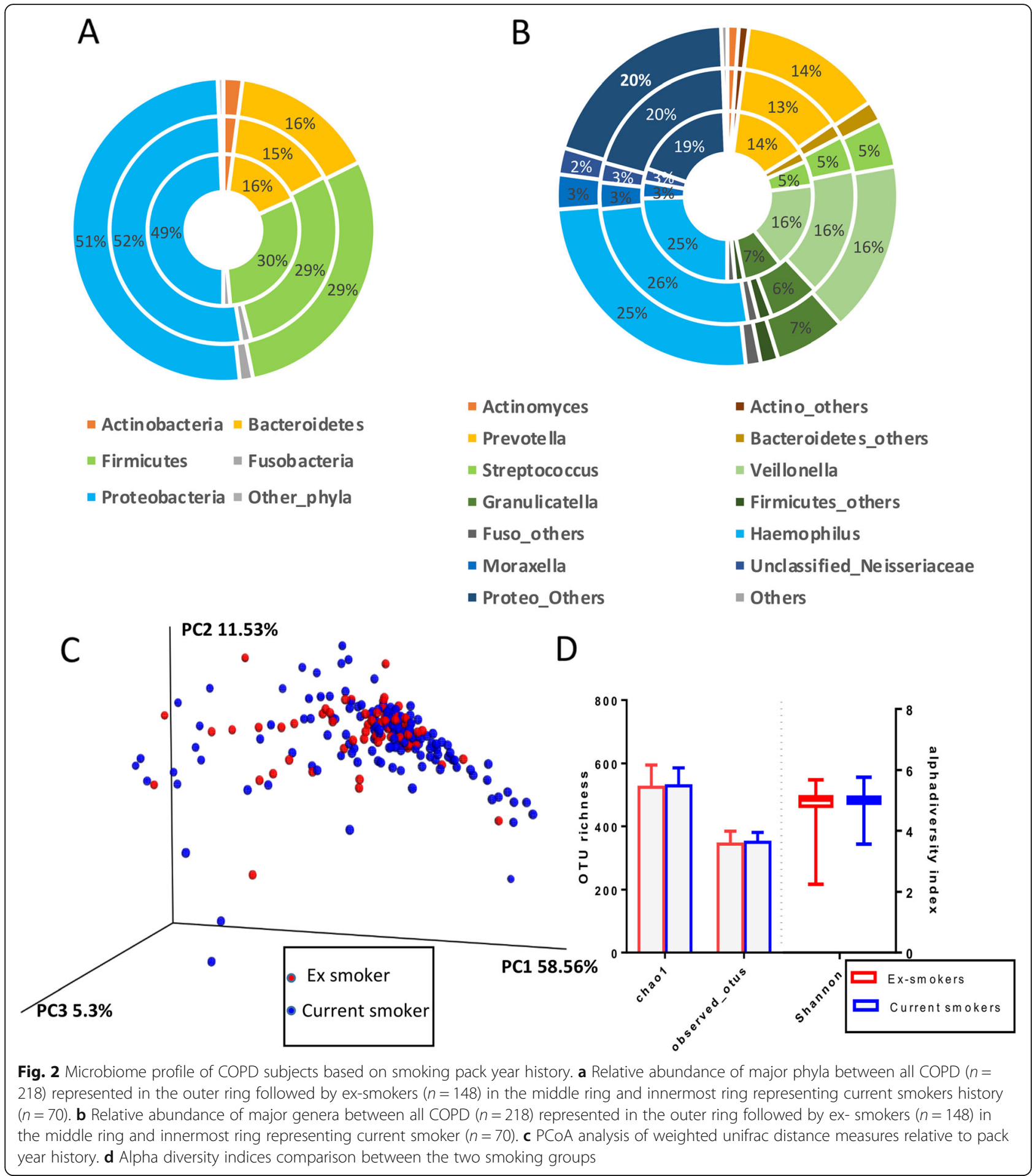

Existing comparative respiratory microbiome data are divided, with some studies reporting an overlapping microbial composition between health and COPD $[3,4,26]$ while others have shown the COPD microbiome to be distinct $[5,6,27]$. These discordant outcomes likely reflect the underlying heterogeneity in COPD groups and small sample sizes of healthy individuals $(<20)$, undermining the strength of these studies [3-6, 26, 27]. However, similar to our observation, higher levels of Proteobacteria, especially Haemophilus, in COPD [3-5] and relatively higher proportion of Prevotella, Veillonella and Actinomyces species in health have been observed [5, 6] but differences did not reach significance. Contrary to our observations, most studies have reported similar or a 


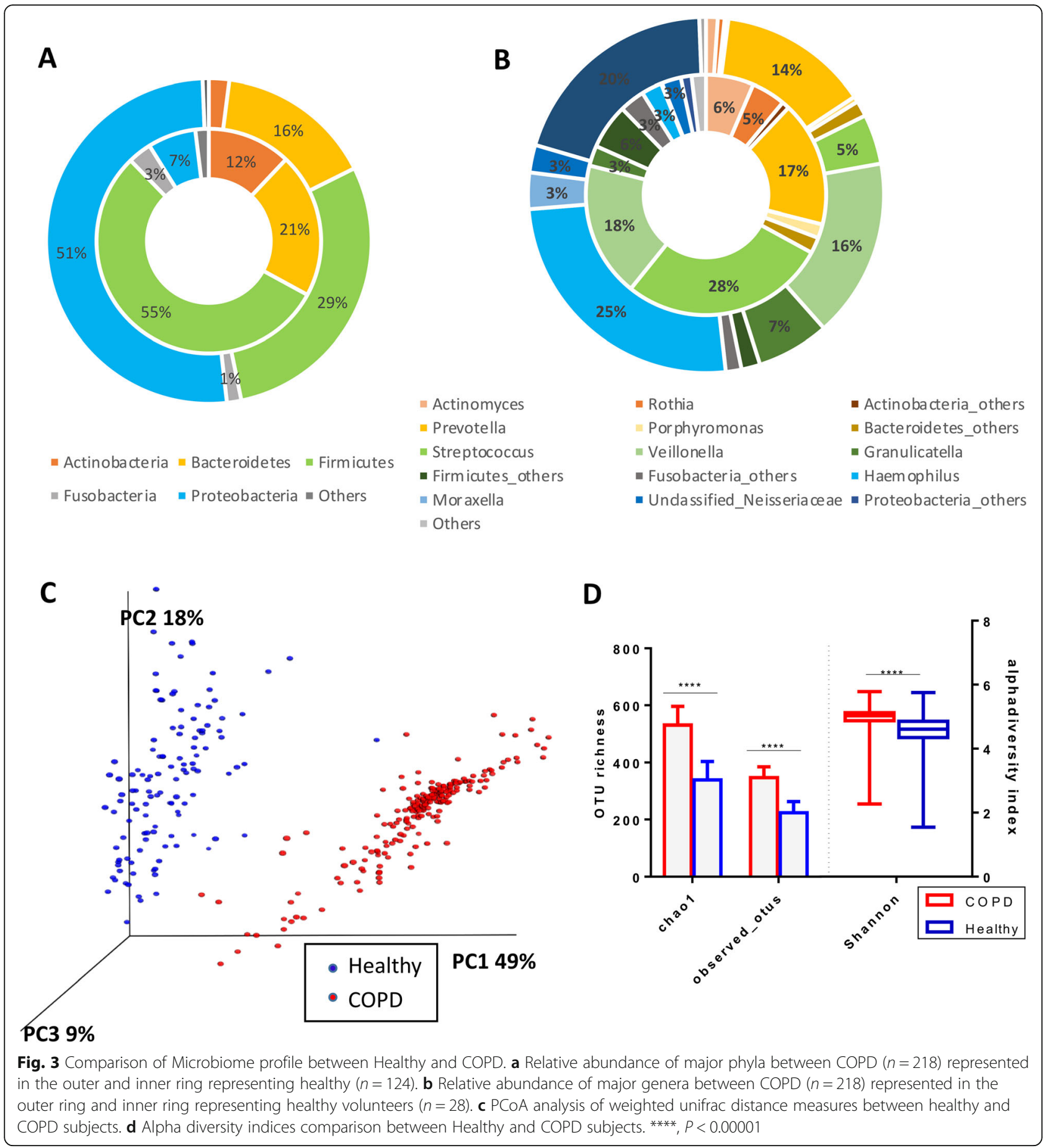

higher abundance of Firmicutes and especially Streptococcus in COPD compared with health $[4,6]$. One reason for this might be that composition varies between samples depending upon the type of treatment received, disease severity and inflammation. Previous studies have shown association between very severe COPD and eosinophilic phenotypes with dominance of Firmicutes, while Proteobacteria are predominant in moderate
COPD and the bacterial related phenotype $[9,28]$. The COPD cohort analysed here was mainly of moderate-tosevere severity with high neutrophil counts.

Contrary to most studies, we found a higher alpha diversity in COPD compared to health $[3,6]$. Although the COPD sample reads were reanalysed with the healthy at a normalised sequence depth, they were sequenced as part of COPDMAP study which involved a much larger 


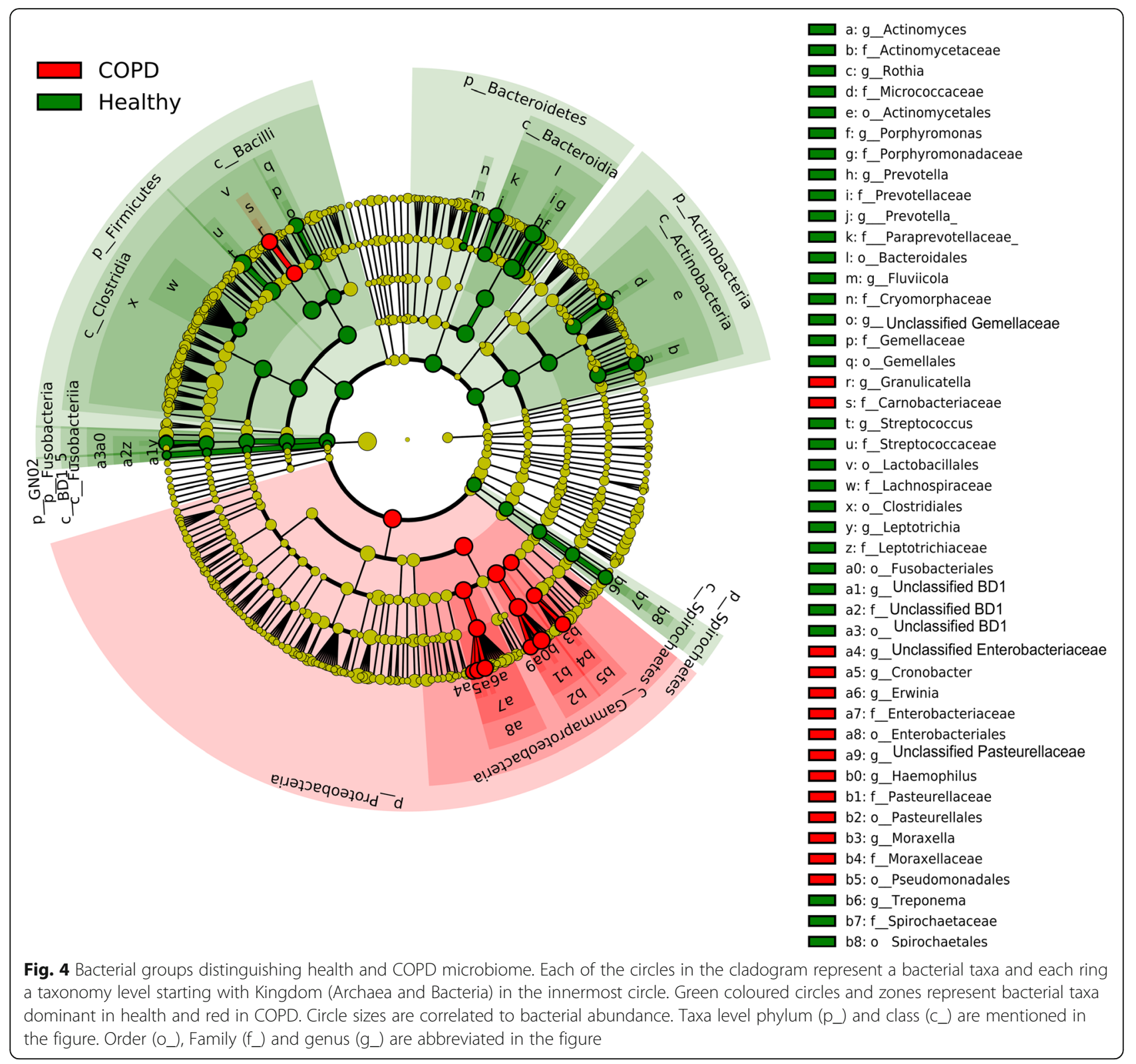

sample size [13], including different disease stages, and this may have contributed higher COPD diversity. Moreover, our COPD cohort was older than the healthy group and from moderate to severe GOLD stage. Higher diversity has been associated with both increasing disease severity and age in COPD [4, 27, 29].

Although a strong association exists between smoking and both airway inflammation and COPD, the determinants for developing COPD in smokers are not clear. We hypothesised that smoking associated microbiome changes in health would help in understanding the role of microbes in transition from health towards COPD. Consistent with previous studies, we found no significant difference between the microbiome of smokers with $\geq 10 \mathrm{PY}$ history and the $<10$ PY group $[3,4,6,30]$.
However, similar to Morris and colleagues [30], a trend towards lower proportions of both Bacteroidetes and Proteobacteria in smokers with $\geq 10 P Y$ history was observed, suggesting subtle effects of smoking on the airway microbiome. Other pathological factors may therefore be important in shaping the microbiome in COPD. Hypoxia and chronic systemic inflammation related factors, which are features of COPD, have been reported to be associated with the airway microbiome [31] and may be relevant to the differences observed in our COPD cohort.

PICRUSt analysis showed relatively higher lipopolysaccharide biosynthesis products in COPD. Lipopolysaccharides are present in the outer membrane of Proteobacteria and together with pathogen-associated 
Table 2 OTU groups that distinguish the Healthy and the COPD subjects

\begin{tabular}{|c|c|c|c|c|c|}
\hline OTU & Taxonomy & $\begin{array}{l}\text { Healthy } \\
\text { mean }\end{array}$ & $\begin{array}{l}\mathrm{COPD}_{-} \\
\text {mean }\end{array}$ & $\begin{array}{l}\text { FDR } \\
\mathrm{P}\end{array}$ & $\begin{array}{l}\text { Bonferroni_ } \\
\text { P }\end{array}$ \\
\hline 579,608 & Firmicutes|Bacilli|Lactobacillales|Streptococcaceae|Streptococcus & 658 & 88 & 0.001 & 0.020 \\
\hline 787,709 & Actinobacteria|Actinobacteria|Actinomycetales|Actinomycetaceae|Actinomyces & 363 & 64 & 0.001 & 0.020 \\
\hline $\begin{array}{l}1,078 \\
207\end{array}$ & Firmicutes|Bacilli|Lactobacillales|Streptococcaceae|Streptococcus & 7 & 184 & 0.001 & 0.020 \\
\hline 865,469 & Proteobacteria|Gammaproteobacteria|Pasteurellales|Pasteurellaceae|Haemophilus & 297 & 2150 & 0.001 & 0.020 \\
\hline 585,419 & Firmicutes|Clostridia|Clostridiales|Veillonellaceae|Veillonella & 1611 & 1214 & 0.001 & 0.020 \\
\hline $\begin{array}{l}1,083 \\
037\end{array}$ & Proteobacteria|Gammaproteobacteria|Pseudomonadales|Moraxellaceae|Moraxella & 12 & 345 & 0.001 & 0.020 \\
\hline 968,954 & Firmicutes|Bacilli|Lactobacillales|Streptococcaceae|Streptococcus & 906 & 2 & 0.001 & 0.020 \\
\hline 932,696 & Proteobacteria|Gammaproteobacteria|Enterobacteriales|Enterobacteriaceae|Erwinia & 0 & 782 & 0.001 & 0.020 \\
\hline 579,924 & Proteobacteria|Gammaproteobacteria|Pasteurellales|Pasteurellaceae| & 2 & 210 & 0.001 & 0.020 \\
\hline $\begin{array}{l}1,083 \\
194\end{array}$ & Firmicutes|Bacilli|Lactobacillales|Streptococcaceae|Streptococcus & 1290 & 5 & 0.001 & 0.020 \\
\hline $\begin{array}{l}1,027 \\
587\end{array}$ & Firmicutes|Bacilli|Lactobacillales|Carnobacteriaceae|Granulicatella & 57 & 702 & 0.001 & 0.020 \\
\hline $\begin{array}{l}1,017 \\
181\end{array}$ & Actinobacteria|Actinobacteria|Actinomycetales|Micrococcaceae|Rothia & 488 & 63 & 0.001 & 0.020 \\
\hline 667,570 & Proteobacteria|Gammaproteobacteria|Enterobacteriales|Enterobacteriaceae|Cronobacter & 0 & 702 & 0.001 & 0.020 \\
\hline 935,742 & Bacteroidetes|Bacteroidia|Bacteroidales|Prevotellaceae|Prevotella & 298 & 101 & 0.001 & 0.020 \\
\hline 342,427 & Firmicutes|Clostridia|Clostridiales|Veillonellaceae|Veillonella & 44 & 284 & 0.001 & 0.020 \\
\hline
\end{tabular}

Non-parametric t-test was performed on rarefied OTU table at 11,000 reds with OTUs that were observed in minimum $25 \%$ of the total number of samples and had a minimum contribution of $1 \%$ of the total reads

molecular patterns, induce strong and damaging proinflammatory responses. In keeping with this, our previous study showed that sputum chemokine interleukin-8, known to play a key role in COPD inflammation, is positively correlated with Haemophilus and Moraxella, suggesting these bacteria trigger the excessive production of this chemokine [9]. Moreover, Haemophilus has been implicated in a dysbiotic role by co-inclusion of its related phylotypes and depletion of Firmicutes, Bacteroidetes and Actinobacteria that are involved in pathways for production of anti-inflammatory compounds $[8,9]$.

Although antibiotic treatment has been associated with suppression of Proteobacteria in COPD [8, 9], it is not true for all cases [10]. With the increasing urgency for effective antibiotic stewardship, research is needed to better understand the impact of both acute and long term antimicrobial therapy on the COPD microbiome. In this respect, alternate therapeutic strategies such as $H$. influenzae vaccination, or highly selective antimicrobial approaches such as phage therapy may effectively reverse some dysbiotic with prognostic benefit.

A limitation of this study is that the lung microbiome has been analysed from sputum samples which can be contaminated with the microbiome of the oropharynx. However, we emphasise that this effect will have been limited by sputum plug selection for the analysis. We did not perform longitudinal sampling to demonstrate reproducibility of the sputum microbiome over time in healthy participants. For COPD we have previously demonstrated that the sputum microbiome is comparable between time-points when sampling at their stable state [32]. The effects on the microbiome of using sputum induction as the predominant sampling technique in the healthy control group are also not known, but it is noteworthy that the predominant bacterial constituents of our healthy microbiome are consistent with the respiratory microbiome detected by investigating BAL and bronchial samples reported in previous studies [3, 6]. This suggests that our observations are robust and representative of the bacterial composition of the lung microbiome. A major incentive to work with sputum is its compatibility with routine clinical practice as any findings are therefore more readily translated into established care pathways. In this study we have not characterized the viral and fungal communities, and this will be important to understand their role in health and disease.

\section{Conclusions}

In summary, clear and significant differences exist between the lung microbiome in health and COPD, with dysbiosis in COPD characterised by increased abundance of Proteobacteria especially Haemophilus. The changes observed in COPD are distinct from the microbiome in smokers without COPD, suggesting an association between airway damage and dysbiosis. The 


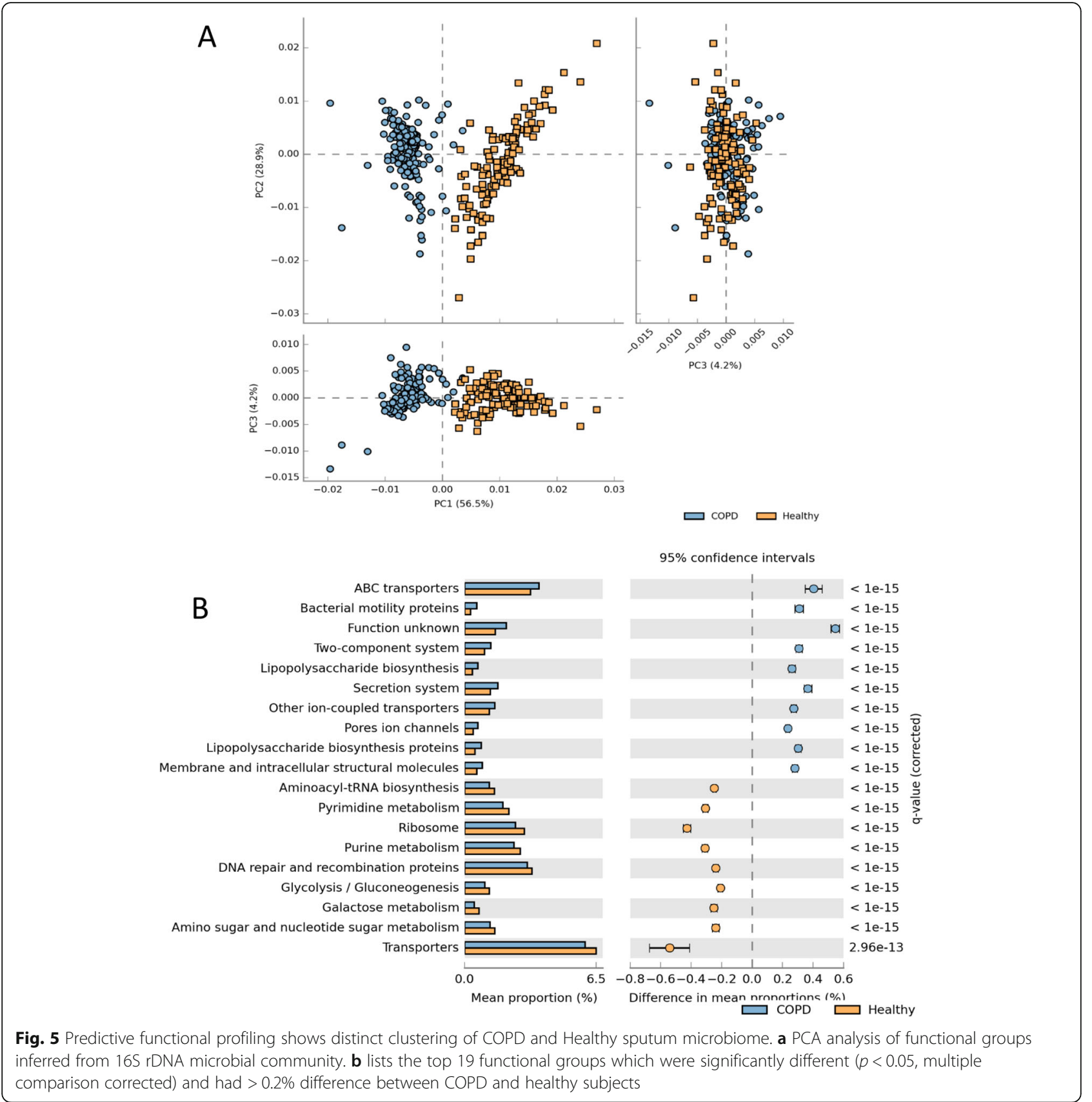

pathogenesis and pathological significance of dysbiosis in COPD remains unclear. Longitudinal studies are needed to determine whether, and to what extent, the onset and progression of COPD are attributable to an altered lung microbiome.

\section{Supplementary information}

Supplementary information accompanies this paper at https://doi.org/10. 1186/s12931-020-01448-3.

\section{Additional file 1}

\section{Abbreviations}

COPD: Chronic obstructive pulmonary Disease; EXCEED: Extended Cohort for E-health, Environment and DNA; KW: Kruksal-Wallis; MaAsLin: Multivariate analysis by linear models; OTU: Operational taxonomic unit; PY: Pack year smoking history; PCOA: Principal coordinate analysis; PERM

ANOVA: Permutation multivariate analysis of variance; SEM: Standard error mean; LefSe: Linear discriminant effect size

\section{Acknowledgements}

The EXCEED study gratefully acknowledges the support of all participants and staff who have contributed to the study. The study has been supported by the University of Leicester, Leicester City Council, the NIHR Leicester Biomedical Research Centre, the NIHR Clinical Research Network East Midlands, and the Medical Research Council (grant G0902313 to MDT) and the Wellcome Trust (grant 202849 to MDT). 


\section{Authors' contributions}

$\mathrm{KH}$ undertook the data analysis and statistical analysis. LG and VM undertook patient recruitment, data collection, and were involved in data analysis. KH and MRB led the microbiological assessments. Samples and analyses were contributed by LG, CJ, NFR and MT (EXCEED), and ZW, JRB, DS, JAW, GCD, BEM and RTS (COPDMAP) study. RF and AJW were responsible for data management. CEB led the design of the study, data collection, data interpretation, data analysis and had full access to the data and was responsible for the integrity of the data and final decision to submit. All the authors contributed to drafting the manuscript or revising it critically for important intellectual content; all authors gave final approval of the version to be published; and all authors agreed to be accountable for all aspects of the work in ensuring that questions related to the accuracy or integrity of any part of the work are appropriately investigated and resolved.

\section{Funding}

This study was part supported by the MRC (COPDMAP), National Institute for Health Research Leicester Biomedical Research Centre, UK, AirPROM (FP7270194). MDT is in receipt of a Wellcome Trust Investigator Award (202849). CJ holds a MRC Clinical Research Training Fellowship (MR/P00167X/1). The views expressed are those of the author(s) and not necessarily those of the $\mathrm{NHS}$, the NIHR or the Department of Health.

\section{Availability of data and materials}

Exceed healthy sample 16 rRNA gene sequence reads are deposited at the National Centre for Biotechnology Information (SRA accession: PRJNA491861) https://www.ncbi.nlm.nih.gov/bioproject/PRJNA491861/.COPDMAP 16S rRNA gene sequence data are deposited at the National Centre for Biotechnology Information Sequence Read Archive (SRP102480) and associated data available from (https://www.ncbi.nlm.nih.gov/search/all/?term=SRP102480).

\section{Ethics approval and consent to participate}

All patients provided written informed consent using protocols approved by the local Ethics Committees at each site (London- 11/L0/1630; Manchester10/H/1003/108; Leicester- 07/H0406/157) for COPDMAP study and the EXCEED study ethics number is: 13/EM/0226.

\section{Consent for publication}

Not applicable.

\section{Competing interests}

$K H, L G, Z W, V M, M Y R, R C F, N F R$, AJW, AJB, MRB have nothing to declare. JRB, BEM and RTS are employees and shareholders of GSK; GCD reports grants and personal fees from Astrazeneca and grants from Micom Itd and American Thoracic Society; MDT reports grants from GSK and other from Orion; JAW reports grants from GSK, grants from Johnson and Johnson, other from Novartis, other from Boehringer Ingelheim, other from Astra Zeneca, other from GSK, grants from GSK, grants from Astra Zeneca, grants from Boehringer Ingelheim, grants from Novartis; DS reports grants and personal fees from GlaxoSmithKline, grants and personal fees from AstraZeneca, grants and personal fees from Boehringer Ingleheim, grants and personal fees from Chiesi, personal fees from Cipla, personal fees from Genentech, grants and personal fees from Glenmark, grants and personal fees from Menarini, grants and personal fees from Mundipharma, grants and personal fees from Novartis, personal fees from Peptinnovate, grants and personal fees from Pfizer, grants and personal fees from Pulmatrix, grants and personal fees from Therevance, grants and personal fees from Verona; CEB reports grants and personal fees from GSK, grants and personal fees from Novartis, grants and personal fees from Genentech, grants and personal fees from Chiesi, personal fees from Sanofi/Regeneron, grants and personal fees from $4 \mathrm{DPharma}$, grants and personal fees from $\mathrm{Bl}$, grants and personal fees from Mologics, grants and personal fees from Gossamer, grants and personal fees from AZ/Medlmmune, grants and personal fees from TEVA, outside the submitted work. CEB reports grants from MRC COPDMAP, grants from AirPROM (FP7-270194), during the conduct of the study.

\section{Author details}

'Institute for Lung Health, NIHR, BRC, Department of Respiratory Sciences, College of Life Sciences, University of Leicester, Leicester LE1 7RH, UK. ${ }^{2}$ Institute of Ecological Science, School of Life Science, South China Normal University, Guangzhou 510631, China. ${ }^{3}$ Department of Health Sciences, NIHR,
BRC, University of Leicester, Leicester LE1 7RH, UK. ${ }^{4}$ Global Medical, GSK, Collegeville, PA 19426, USA. ${ }^{5}$ Department of Genetics, University of Leicester, Leicester LE1 7RH, UK. ' University of Manchester and University Hospital of South Manchester, Manchester M23 9QZ, UK. ${ }^{7}$ National Heart and Lung Institute, Imperial College London, London SW3 6NP, UK. ${ }^{8}$ Computational Biology, Human Genetics, Research and Development (R\&D), GlaxoSmithKline (GSK), Collegeville, PA 19426, USA. ${ }^{9}$ Institute for Lung Health, University Hospitals of Leicester, Groby Road, Leicester LE3 9QP, UK.

\section{Received: 9 April 2020 Accepted: 8 July 2020}

Published online: 14 July 2020

\section{References}

1. Sethi S, Murphy TF. Infection in the pathogenesis and course of chronic obstructive pulmonary disease. N Engl J Med. 2008;359(22):2355-65.

2. Dickson RP, Martinez FJ, Huffnagle GB. The role of the microbiome in exacerbations of chronic lung diseases. Lancet. 2014;384(9944):691-702.

3. Erb-Downward JR, Thompson DL, Han MK, Freeman CM, McCloskey L, Schmidt LA, et al. Analysis of the lung microbiome in the "healthy" smoker and in COPD. PLoS One. 2011;6(2):e16384.

4. Pragman AA, Kim HB, Reilly CS, Wendt C, Isaacson RE. The lung microbiome in moderate and severe chronic obstructive pulmonary disease. PLoS One. 2012;7(10):e47305.

5. Hilty M, Burke C, Pedro H, Cardenas P, Bush A, Bossley C, et al. Disordered microbial communities in asthmatic airways. PLoS One. 2010;5(1):e8578.

6. Einarsson GG, Comer DM, Mcllreavey L, Parkhill J, Ennis M, Tunney MM, et al. Community dynamics and the lower airway microbiota in stable chronic obstructive pulmonary disease, smokers and healthy non-smokers. Thorax. 2016;71(9):795-803.

7. Cabrera-Rubio R, Garcia-Núñez M, Setó L, Antó JM, Moya A, Monsó E, et al. Microbiome diversity in the bronchial tracts of patients with chronic obstructive pulmonary disease. J Clin Microbiol. 2012;50(11):3562-8.

8. Huang YJ, Sethi S, Murphy T, Nariya S, Boushey HA, Lynch SV. Airway microbiome dynamics in exacerbations of chronic obstructive pulmonary disease. J Clin Microbiol. 2014;52:2813-23.

9. Wang Z, Bafadhel M, Haldar K, Spivak A, Mayhew D, Miller BE, et al. Lung microbiome dynamics in COPD exacerbations. Eur Respir J. 2016;47(4):108292.

10. Haldar K, Bafadhel M, Lau K, Berg A, Kwambana B, Kebadze T, et al. Microbiome balance in sputum determined by PCR stratifies COPD exacerbations and shows potential for selective use of antibiotics. PLoS One. 2017;12(8):e0182833.

11. Charlson ES, Chen J, Custers-Allen R, Bittinger K, Li H, Sinha R, et al. Disordered microbial communities in the upper respiratory tract of cigarette smokers. PLoS One. 2010;5(12):e15216.

12. Johannessen A, Omenaas ER, Bakke PS, Gulsvik A. Implications of reversibility testing on prevalence and risk factors for chronic obstructive pulmonary disease: a community study. Thorax. 2005;60(10):842-7.

13. Wang Z, Singh R, Miller BE, Tal-Singer R, Van Horn S, Tomsho L, et al. Sputum microbiome temporal variability and dysbiosis in chronic obstructive pulmonary disease exacerbations: an analysis of the COPDMAP study. Thorax. 2018;73(4):331-8.

14. Kuczynski J, Stombaugh J, Walters WA, González A, Caporaso JG, Knight R. Using QIIME to analyze 16S rRNA gene sequences from microbial communities. Curr Protoc Microbiol. 2012;27:1E-5.

15. Bolger AM, Lohse $\mathrm{M}$, Usadel B. Trimmomatic: a flexible trimmer for Illumina sequence data. Bioinformatics. 2014;30(15):2114-20.

16. Aronesty E. Command-line tools for processing biological sequencing data. 2011. Available from: https://github.com/ExpressionAnalysis/ea-utils.

17. Edgar RC, Haas BJ, Clemente JC, Quince C, Knight R. UCHIME improves sensitivity and speed of chimera detection. Bioinformatics. 2011;27(16): 2194-200.

18. Wang Q, Garrity GM, Tiedje JM, Cole JR. Naive Bayesian classifier for rapid assignment of rRNA sequences into the new bacterial taxonomy. Appl Environ Microbiol. 2007;73(16):5261-7.

19. Lozupone C, Knight R. UniFrac: a new phylogenetic method for comparing microbial communities. Appl Environ Microbiol. 2005;71(12):8228-35.

20. Langille MGI, Zaneveld J, Caporaso JG, McDonald D, Knights D, Reyes JA, et al. Predictive functional profiling of microbial communities using 165 rRNA marker gene sequences. Nat Biotech. 2013;31(9):814-21. 
21. Parks DH, Tyson GW, Hugenholtz P, Beiko RG. STAMP: statistical analysis of taxonomic and functional profiles. Bioinformatics. 2014;30(21):3123-4.

22. Anderson MJ. A new method for non-parametric multivariate analysis of variance. Aust Ecol. 2001;26(1):32-46.

23. Segata N, lzard J, Waldron L, Gevers D, Miropolsky L, Garrett WS, et al. Metagenomic biomarker discovery and explanation. Genome Biol. 2011; 12(6):R60.

24. Bergen B, Endres S, Engel A, Zark M, Dittmar T, Sommer U, et al, Acidification and warming affect prominent bacteria in two seasonal phytoplankton bloom mesocosms. Environ Microbiol. 2016;18(12):4579-95.

25. Morgan XC, Tickle TL, Sokol H, Gevers D, Devaney KL, Ward DV, et al. Dysfunction of the intestinal microbiome in inflammatory bowel disease and treatment. Genome Biol. 2012;13(9):R79.

26. Diao W, Shen N, Du Y, Erb-Downward JR, Sun X, Guo C, et al. Symptomrelated sputum microbiota in stable chronic obstructive pulmonary disease. Int J Chronic Obstruct Pulmon Dis. 2018;13:2289-99.

27. Sze MA, Dimitriu PA, Hayashi S, Elliott WM, McDonough JE, Gosselink JV, et al. The lung tissue microbiome in chronic obstructive pulmonary disease. Am J Respir Crit Care Med. 2012;185(10):1073-80.

28. Pragman AA, Lyu T, Baller JA, Gould TJ, Kelly RF, Reilly CS, et al. The lung tissue microbiota of mild and moderate chronic obstructive pulmonary disease. Microbiome. 2018;6:7.

29. Pragman AA, Kim HB, Reilly CS, Wendt C, Isaacson RE. Chronic obstructive pulmonary disease lung microbiota diversity may be mediated by age or inhaled corticosteroid use. J Clin Microbiol. 2015;53:1050.

30. Morris A, Beck JM, Schloss PD, Campbell TB, Crothers K, Curtis JL, et al. Comparison of the respiratory microbiome in healthy nonsmokers and smokers. Am J Respir Crit Care Med. 2013;187(10):1067-75.

31. Shukla SD, Walters EH, Simpson JL, Keely S, Wark PAB, O'Toole RF, et al. Hypoxia-inducible factor and bacterial infections in chronic obstructive pulmonary disease. Respirology. 2020;25(1):53-63.

32. Bafadhel M, Haldar K, Barker B, Patel H, Mistry V, Barer MR, et al. Airway bacteria measured by quantitative polymerase chain reaction and culture in patients with stable COPD: relationship with neutrophilic airway inflammation, exacerbation frequency, and lung function. Int J Chron Obstruct Pulmon Dis. 2015;10:1075-83.

\section{Publisher's Note}

Springer Nature remains neutral with regard to jurisdictional claims in published maps and institutional affiliations.

Ready to submit your research? Choose BMC and benefit from:

- fast, convenient online submission

- thorough peer review by experienced researchers in your field

- rapid publication on acceptance

- support for research data, including large and complex data types

- gold Open Access which fosters wider collaboration and increased citations

- maximum visibility for your research: over $100 \mathrm{M}$ website views per year

At $\mathrm{BMC}$, research is always in progress.

Learn more biomedcentral.com/submissions 